\title{
Capillaroscopic patterns in patients with systemic sclerosis, psoriasis and alopecia and their correlations with serum concentrations of several angiogenic markers
}

\begin{abstract}
Introduction. Capillaroscopy is a non-invasive imaging method that allows cutaneous microcirculation to be analyzed. During the last decades, a diagnostic and prognostic potential of nailfold capillaroscopy (NVC) has been gaining increasing appreciation. The main indications include Raynaud phenomenon and scleroderma spectrum diseases, however the usefulness of this technique is also suggested in a variety of non-rheumatic diseases.

Aim. To assess capillaroscopic patterns in systemic scleroderma (SSc), psoriasis (PV), psoriatic arthritis (PsA), alopecia areata (AA) and androgenetic alopecia. To evaluate serum levels of several endothelial and angiogenic markers, and their relation to capillaroscopic pattern.

Material and methods. There were evaluated 295 patients with systemic scleroderma (SSc), psoriasis (PV), psoriatic arthritis (PsA), alopecia areata (AA) and androgenetic alopecia, as well as age- and sex-matched controls, were examined. In each subject, $\mathrm{NVC}$ was performed and serum concentration levels of several angiogenic markers.

Results. In SSc three NVC patterns: early, active and late were distinguished. Angiopoietin-2 concentrations were higher and andothelial microparticles were lower in patients with late NVC pattern. We found several differences between the NVC pattern in PV and PsA. No correlations between NVC pattern and serum levels of angiogenic markers were revealed. In AA, we distinguished both normal and abnormal NVC patterns, although the normal patterns were more frequent. Branching capillaries and features of neoformation were often present in patients with the abnormal pattern. In androgenetic alopecia, the normal NVC pattern was most frequently present, however, we found several statistically significant capillarosopic alterations, like branching capillaries, features of neoformation and altered distribution of capillaries.

Discussion and Conclusions. Serum levels of Ang-2 and EMPs may reflect capillary damage in SSc. NVC pattern varies between PV and PsA patients. The presence of abnormal NVC patterns in alopecia patients might show the role of disturbances in microcirculation in the diseases. Further studies are required to confirm the hypothesis.
\end{abstract}

Keywords: capillaroscopy, nailfold videocapillaroscopy, angiogenesis, systemic scleroderma, psoriasis, psoriatic arthritis, alopecia areata, androgenetic alopecia.

DOI: $10.1515 /$ pjph-2015-0024

\section{INTRODUCTION}

Capillaroscopy (intravital capillary microscopy) is a noninvasive imaging technique, used for an in vivo analysis of cutaneous microcirculation, as well as quantifying some of its parameters. Capillaroscopy is one of the safest and quickest diagnostic systems used for detection and monitoring of morphological alterations of microvessels. It is so useful because it reveals even minute changes. It can be performed with various optical instruments (Figure 1A) and principally, it is carried out using a nailfold capillary bed. This is due to the fact that the capillaries in this area are arranged in a parallel fashion with respect to the skin surface, which means that a large part of their loops can be observed (Figure 1B). Despite that, the tool that was introduced recently, namely videocapillaroscopy (VC), has some further advantages. A 200x magnification optical contact probe is connected to a mini video recorder (Figure 1C,D), which significantly improves the quality and allows for an analysis of microcirculation at all cutaneous sites (nailfold videocapillaroscopy - NVC and out-of-nailfold videocapillaroscopy; Figure 1E) to be performed [1].

During the last decades, there has been a widespread interest in NVC and its diagnostic and prognostic power is being increasingly appreciated. Still, the main indication for performing NVC is the differentiation between primary and secondary Raynaud phenomenon (RP). However, a potential application of this technique is also suggested in a variety of non-rheumatic diseases in which angiogenic alterations are present, including diabetes mellitus, cardiac syndrome $\mathrm{X}$, arterial hypertension, acromegaly, hyperthyroidism, primary biliary cirrhosis, Crohn's disease, psoriasis, and familial Mediterranean fever [2]. 


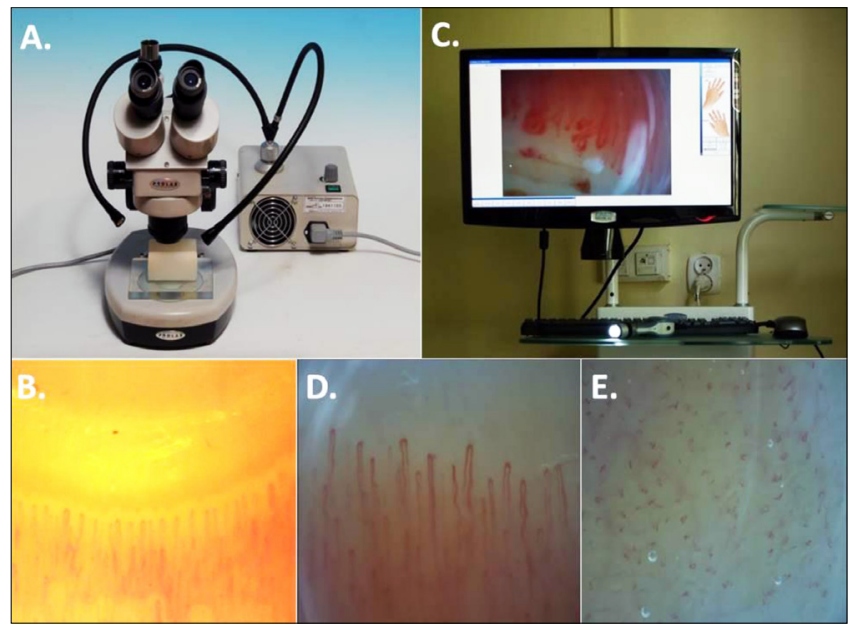

FIGURE 1. A. Stereomicroscope; B. Classic wide-field image of the nailfold capillary bed (magnification, x 50; stereomicroscope); a normal capillaroscopic pattern - capillaries of the distal row have an open hairpin shape, their sizes are homogenous and they are regularly arranged in parallel fashion with respect to the skin surface; the number of capillary loops ranges from 9-13/linear millimetre; C. Videocapillaroscope device; D. A close-up view of normal capillaroscopic pattern of the nailfold (magnification, $x$ 200; videocapillaroscope). The image represents approximately $1.0 \mathrm{~mm}$ across the long axis; E. Out-of-nailfold image of cutaneous capillaries the capillaries tend to run perpendicular to the skin surface; only their apexes are visible (magnification, $x$ 200; videocapillaroscope).

The history of capillaroscopy in the Department of Dermatology began over 20 years ago, with Janusz Urban as a pioneer in this field. In 2009, as the first research center in Poland, we started using the video microscopy equipment (Figure 1C). Since then, we have been focused on intensive studies on cutaneous microcirculation in rheumatic and nonrheumatic disease. We paid special attention to RP, systemic sclerosis $(\mathrm{SSc})$, psoriasis vulgaris $(\mathrm{PV}) /$ psoriatic arthritis (PsA), alopecia areata (AA) and androgenetic alopecia. In each disease, angiogenic alterations may play an important role.

\section{AIM}

The aim of the study was to assess capillaroscopic patterns in SSc, PV, PsA, AA and androgenetic alopecia. Also, we wanted to evaluate serum levels of several endothelial and angiogenic markers, as well as their relation to the capillaroscopic pattern in these diseases.

\section{MATERIAL AND METHODS}

A total of 295 patients and age- and sex- matched healthy controls were enrolled in the study (Table 1). In case of all patients and controls, serum levels of certain angiogenic markers were measured. All measurements were performed by ELISA, except from endothelial microparticles (EMPs), which were identified by fluorocytometry in platelet-poor plasma according to the procedures described by Jimenez et al. [3] and Bernal-Mizrachi et al. [4]. NVC was performed in patients with SSc, PV, PsA, AA and androgenetic alopecia, as well as in matched healthy controls in a blind manner, using a videocapillaroscope (VideoCap 3.0, DS Medica, Milano, Italy) by one researcher, basing on the instructions described previously [1]. Patients with SSc were classified according to Cutolo et al. as those with an early, active and late capillaroscopic pattern [1] (Figure 2).

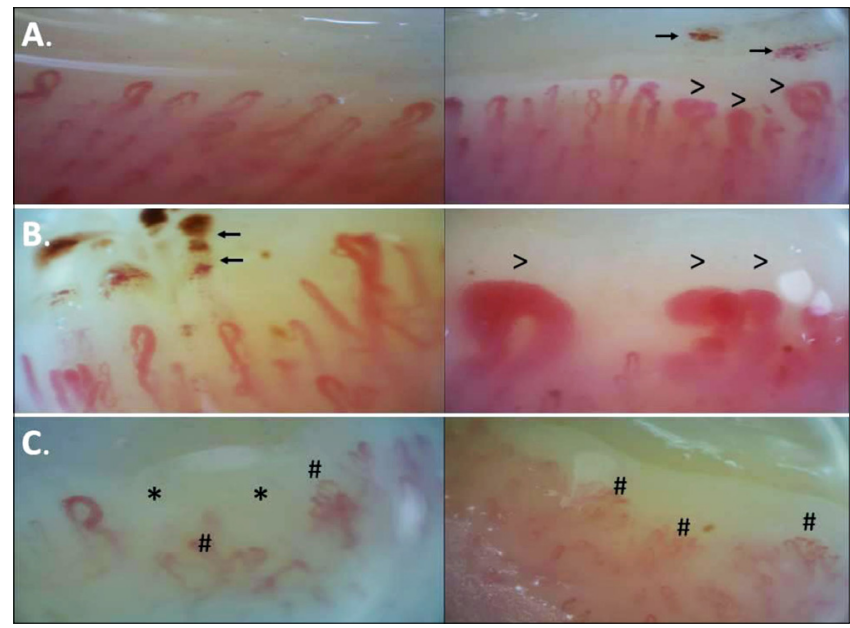

FIGURE 2. Capillaroscopic patterns of microangiopathy in systemic sclerosis. A. "Early" scleroderma pattern: few giant capillaries $(>)$, few microhaemorrhages $(\rightarrow)$, no evident loss of capillaries, and relatively well-preserved capillary distribution; B. "Active" scleroderma pattern: frequent giant capillaries $(>)$, frequent microhaemorrhages $(\rightarrow)$, moderate loss of capillaries, absent or mildly ramified capillaries, moderate derangement of capillary array; C. "Late" scleroderma pattern: irregular enlargement of capillaries, almost absent giant capillaries and microhaemorrhages, severe loss of capillaries with marked avascular areas (*), ramified/ bushy (neoangiogenetic) loops (\#), severe disorganisation of capillary distribution.

\section{RESULTS}

Systemic sclerosis. An early capillaroscopic pattern was found in $14(29.79 \%)$, active pattern in $14(29.79 \%)$ and late pattern in $19(40.42 \%)$ of $47 \mathrm{SSc}$ patients. No significant differences in the levels of Ang-1 were found between the three capillaroscopy groups of SSc patients. In contrast, serum concentrations of Ang-2 were significantly increased in patients with the late pattern, when compared against those SSc patients, who had capillaroscopic changes typical of active pattern $(\mathrm{p}<0.05)$. Serum levels of Ang-2 in SSc subjects with late capillaroscopic pattern were also higher, but not significantly different than in patients with early changes $(\mathrm{p}>0.05)[5]$.

Serum Angiopoietin-1 (Ang-1) was significantly decreased $(\mathrm{p}<0,01)$, while serum Angiopoietin-2 (Ang-2) was substantially elevated $(\mathrm{p}<0,01)$ in patients with SSc as compared with healthy controls [5].

TABLE 1. Number of participants in our research and parameters evaluated in each of studied disease (Ang-1: Angiopoietin-1, Ang-2: Angiopoietin-2, EMPs: endothelial microparticles, PIGF: placental growth factor, sVEGFR-1: Vascular Endothelial Growth Factor Receptor-1, VEGF-A: Vascular Endothelial Growth Factor A, HGF: hepatocyte growth factor, IGF-1: insulin like growth factor 1).

\begin{tabular}{lccc}
\hline \multicolumn{1}{c}{ Disease } & $\begin{array}{c}\text { Number of } \\
\text { patients }\end{array}$ & $\begin{array}{c}\text { Number of } \\
\text { healthy controls }\end{array}$ & $\begin{array}{c}\text { Parameters } \\
\text { measured }\end{array}$ \\
\hline $\begin{array}{l}\text { Systemic } \\
\text { scleroderma }\end{array}$ & 47 & 27 & Ang-1, Ang-2, EMPs \\
\hline $\begin{array}{l}\text { Psoriasis/ } \\
\text { Psoriatic } \\
\text { arthritis }\end{array}$ & 117 & 30 & $\begin{array}{c}\text { PlGF, sVEGFR-1, } \\
\text { endostatin, } \\
\text { thrombospondin-2 }\end{array}$ \\
\hline $\begin{array}{l}\text { Alopecia } \\
\text { areata }\end{array}$ & 71 & 40 & VEGF-A, HGF, IGF-1, \\
\hline $\begin{array}{l}\text { Androgenetic } \\
\text { alopecia }\end{array}$ & 60 & 40 & VEGF-A, HGF, IGF-1, \\
\hline
\end{tabular}


In the field of EMPs, preliminary study showed that all types of EMPs were significantly elevated in SSc patients as compared with healthy controls. Significant correlations have been observed between levels and phenotypes of EMPs and detailed malformations of nailfold capillaries (ahead of pub). The mean concentration of total EMPs (CD31+/CD42b-) in SSc patients with late pattern in capillaroscopy was significantly lower as compared with SSc patients with early capillarscopic pattern $(p<0.05)$, and tended to be lower as compared with SSc patients with active pattern $(\mathrm{p}=0.1)$, however, we found no significant differences in the concentration levels of any of EMPs' subpopulations (activated/apoptotic), between SSc patients with different capillaroscopic patterns (data unpublished).

Psoriasis. The incidence of tortuous capillaries was significantly higher in the studied psoriatic patients, when compared against the healthy group $(\mathrm{p}=0.018)$. Psoriatic patients presented the following nailfold capillaries: coiled $(24.32 \%)$, of a "psoriatic plaque" type (15.32\%), "M"-shaped (18.92\%), bushy $(0.9 \%)$, branching $(10.81 \%)$, all of which were not observed in the controls. In the patients with PsA, the subpapillary venular plexus was visible significantly more often than in the PV patients. Moreover, in the PsA patients reported a decreased number of nailfold capillaries, as well as the presence of thin and frail capillaries, significantly more common than in healthy subjects.

Statistically significant activity of PlGF, endostatin and thrombospondin in psoriatic patients, in comparison with healthy subjects, was found $(\mathrm{p}<0.000001)$. No statistical difference in the serum sVEGF activity between the psoriatic patients and healthy subjects was observed $(p=0.175)$. Statistical analysis of the serum activity of the selected angiogenic markers, i.e. PlGF, sVEGF, endostatin, thrombospondin 2 and four distinguished capillaroscopic patterns (described below) (Figure 3), did not reveal any significant correlations ( $p=0.600 ; p=0.149 ; p=0.673 ; p=0.289$, respectively) (unpublished data).

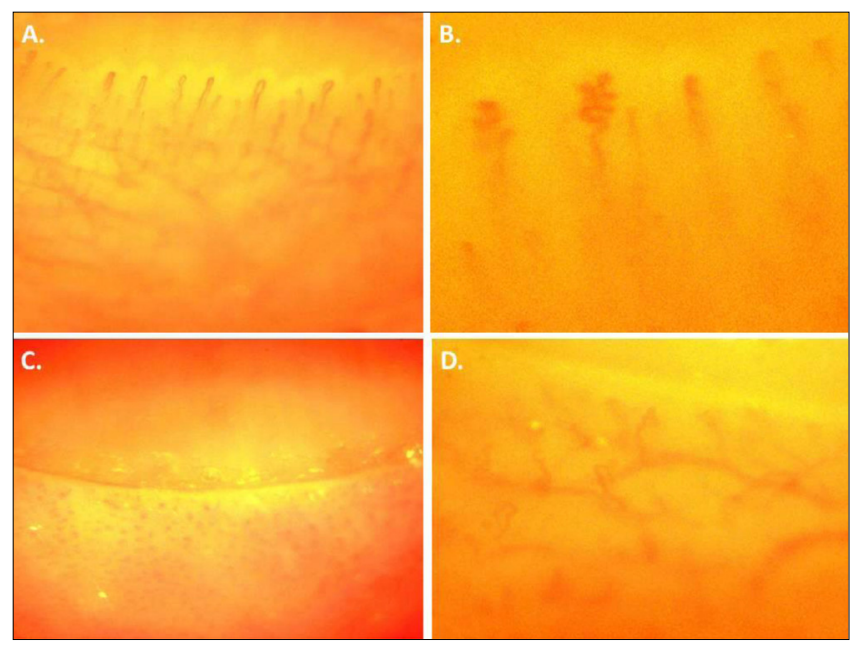

FIGURE 3. Four original types of NVC patterns in psoriatic patients. A. Type I: pink background, "U-shaped" loops, normal distribution; B. Type II: tortuous capillaries; C. Type III: red background, oedema and "plaque-type" capillaries; D. Type IV: pale background, decreased number of capillaries, presence of thin capillaries and well-visible subpapillary venular plexus.
Alopecia areata. Two types of NVC images were distinguished. The most common was the normal pattern (similar to healthy population), present in 41 patients. In the abnormal pattern, which was recognized in 30 subjects, at least 2 NVC alterations in minimum 2 fingers were observed [6]. The NVC images were varied. There was no statistical correlation between the severity of AA and the presence of abnormal NVC images $p=0.233$ [6]. Moreover, the disease activity did not correlate with NVC alterations $(p=0.223)$ (unpublished data).

Among patients with the second type of NVC images, all subjects had tortuous loops (Figure 4). Most patients had multiple tortuous loops with irregular appearance, defined by the presence of multiple crossovers or morphology of the loops described as: "treble clef" loop, "antler" loop, "trefoil" loop as well as meandering appearance with single or multiple crossovers. Compared against the healthy subjects in this group, we observed significantly more often branching capillaries and features of the capillary neoformation, characterized by the presence of thin interconnection between the limbs, or thin branching within one $\operatorname{limb}(\mathrm{p}=0.001$, $\mathrm{p}=0.021$, respectively).

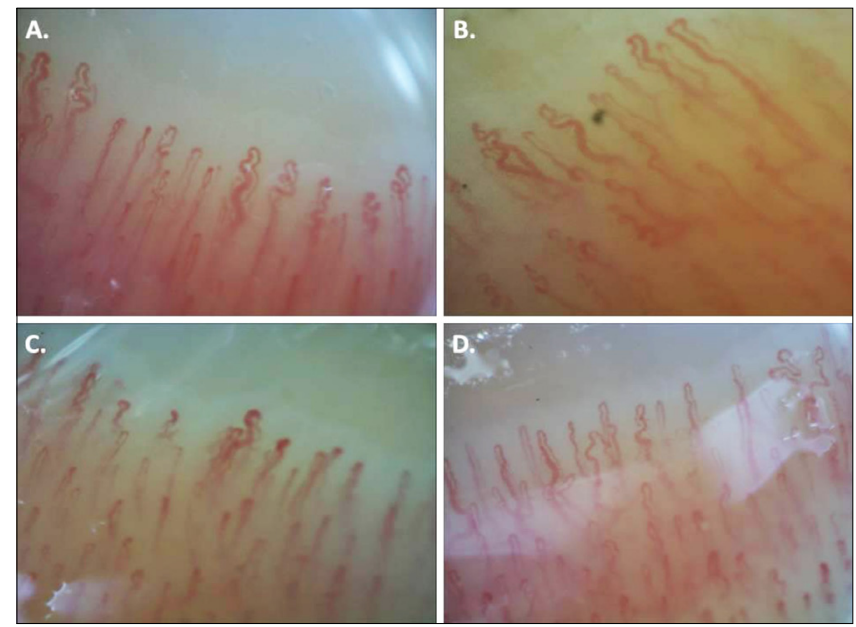

FIGURE 4. Androgenetic alopecia. Second (abnormal) type of NVC images: A. dilatation of efferent limb, features of capillaries neoformation, multiple tortuous loops; B. dilation of efferent limb, features of capillaries neoformation, irregular tortuous loops; C. enlargement of top part of the loop, features of capillaries neoformation, single tortuous loops; D. dilatation of the efferent limb, features of capillaries neoformation, tortuous loops.

There were no significant differences between the presence of NVC alterations in patients with AA and serum concentration of VEGF-A, HGF, IGF-1 and endostatin (respectively $\mathrm{p}=0.779, \mathrm{p}=0.843, \mathrm{p}=0.788, \mathrm{p}=0.530$ ) (unpublished data).

Androgenetic alopecia. Tortuous loops were observed in $98 \%$ of patients with androgenetic alopecia. Among these, $21.6 \%$ had multiple tortuous loops. Branching capillaries and features of neoformation were observed significantly more often respectively in $6.67 \%$ and $18.33 \%$ of study subjects than in a control group. In our observation, the dilation of the efferent limb was found in $10 \%$ of patients. The frequency of these alterations was significantly higher in the studied group than in the control group $(\mathrm{p}<0.017)$. Altered distribution of capillaries was observed significantly more often in the patients with pattern hair loss, when compared to the healthy volunteers $(\mathrm{p}=0.0003)$. 
Based on the results, we distinguished two types of NVC images in the patients with androgenic alopecia. The first, normal pattern, was present in the majority of patients (47-78.3\%). The abnormal NVC images were present in 13 patients. Similarly to AA, NVC images varied, but each patient had at least 2 alterations in at least 2 fingers. All subjects in this group had altered distribution of capillaries and multiple tortuous loops, the majority of which had an irregular appearance. In contrast to the patients with the first type of NVC pattern, or the control group the features of capillary neoformation were shown in majority of patients $(\mathrm{p}=0.002)$ (unpublished data).

There was no statistical correlation between the severity of the hair loss and the presence of abnormal NVC images $(p=0.078)$. Similarly, no significant differences were shown between the presence of NVC alterations and serum concentration of VEGF-A, HGF, IGF-1 and endostatin (respectively $\mathrm{p}=0.346 ; \mathrm{p}=0.456 ; \mathrm{p}=0.542 ; \mathrm{p}=0.836$ ) (unpublished data).

\section{DISCUSSION}

Systemic sclerosis. In 1925 Brown and O'Leary used capillaroscopic analysis for the first time to show in detail the abnormalities that characterize the involvement of the microvasculature in SSc [7]. In 1973 Maricq and LeRoy made the first study describing specific capillaroscopic patterns in SSc, then reclassified by Cutolo et al. in year 2000 into three defined major patterns: early, active and late, each characterized by different proportions of scleroderma-type hallmark parameters: giant capillaries (megacapillaries), microhaemorrhages, ramifications and capillary loss $[8,9]$ (Figure 2).

More than $95 \%$ of SSc patients have specific capillary abnormalities, even in the early stages of the disease, when clinical features are only limited to RP. Therefore, it has been proposed that specific alterations in nailfold capillaries should be included as classification criteria for very early SSc (VEDOSS) [10], as well as the newest classification criteria for SSc, approved by the American College of Rheumatology (ACR) Board of Directors and the European League Against Rheumatism (EULAR) Executive Committee, which replaced previous ARA criteria established in 1980 [11].

The links between NVC and clinical markers of the disease are important issues in SSc. It has been shown that more advanced capillary loss is associated with the development of clinically relevant skin involvement with digital ulcers and poor prognosis [12]. NVC abnormalities might also reflect what is going on in the pulmonary circulation and recent studies suggest that nailfold capillary density reduction is a marker of the presence and severity of pulmonary hypertension (PAH). As a consequence, the early detection of SSc microangiopathy could allow preventive treatment with reduction of morbidity $[13,14]$. Although the role of vascular damage in SSc seems to be important, mechanisms leading to alterations in microcirculation remain unclear. Hence, there is a growing interest and intense recent research on reliable biological markers of endothelial injury and defective angiogenesis in vitro in combination with structural changes in capillary loops observed in vivo.
In our study we described several relationships between pathological changes in nailfold capillary bed in SSc patients and endothelial activation/damage markers as well as angiogenic factors, including angiopoietins and EMPs. Angiopoietins are ligands for the endothelium-specific tyrosine kinase Tie 2 receptor. The role of Ang-1 and -2 has been clarified as they might act alongside VEGF, a key molecule involved in angiogenesis [15]. Angiopoietins are known to be involved in the development, remodelling and stability of blood vessels. It was therefore tempting to hypothesize that altered expression of Ang-1 and -2 might contribute to the pathogenesis of SSc through promoting development of SSc-related vasculopathy [16]. In fact, we found serum concentrations of Ang-2 were significantly increased in patients with the late pattern when compared with those SSc patients who had capillaroscopic changes typical of active pattern. Hence, these observations may suggest that significantly increased levels of Ang-2 in sera of SSc patients reflect vascular injury and might be associated with more advanced capillary damage.

Although we did not observe any differences in Ang-1 levels directly, according to the type of capillaroscopic pattern, it might be concluded that differential expression of Ang-1/Ang-2 might contribute to the pathogenesis of SSc possibly through the contribution to disturbed formation, maturation and integrity of blood vessel [5].

Recently, EMPs have emerged as new markers providing reliable information on endothelium status in various vascular diseases, including rheumatic disorders. Microparticles are a heterogeneous population of small $(0.1-1.0 \mu \mathrm{m})$, membrane-coated vesicles, shed from plasma membrane of different cells, in response to cellular dysfunction and/or injury and circulating in the blood. It needs emphasizing that the EMPs released from activated and apoptotic cells are phenotypically and quantitatively distinct. In general, the activation of endothelial cells without apoptosis, significantly increases expression of inducible antigens on EMPs (e.g., CD62E, CD106, CD54), while processes of apoptosis are reflected by the improved expression of constitutive antigens on EMPs (e.g., CD31, CD105) and increased binding of annexin $\mathrm{V}$ [16].

We have demonstrated that the concentration levels of total circulating EMPs were significantly higher in SSc patients, when compared against healthy controls. Additionally, the mean concentration of total EMPs was significantly lower in SSc patients with late capillaroscopic pattern, as compared against the SSc patients with the early pattern and tended to be lower as compared with active pattern SSc patients. All those capillaroscopic patterns differ, with respect to the severity of capillary loss and the late pattern is being characterized by the most severe avascularization. The early pattern is characterized by relatively well-preserved capillary numbers, and the active pattern - by intermediate changes. Therefore, it could be suggested that the concentration levels of total EMPs are related to the overall number of microvessels in SSc. Indeed, we found out that the levels of total EMPs are inversely correlated to the severity of capillary loss. Thus, our preliminary results suggest that quantity of circulating EMPs might reflect microvascular changes in SSc. Further studies are required to reveal the role of EMPs in the development of microangiopathy in SSc. 
Psoriasis. Angiogenesis is one of the most essential pathophysiological mechanisms in PV. Vascular changes appear at an early stage of psoriatic plaque development before the occurrence of the clinical and histopathological features of epidermis hyperplasia. They are due to the endothelial cells proliferation which leads to the elongation of the venous part of capillaries in the dermal papilla. The arterial limb becomes proportionally shorter and nearly all the loop is of the venular nature ("venulization" phenomenon) [17]. Moreover, within the elongated, tortuous, dilated intrapapillary capillaries the gaps in the endothelium are present with an increased blood flow and leakage of albumin and other blood proteins to the surrounding tissue $[17,18]$. In PV this balance is disturbed, which may be reflected in the tissue and serum pro- and anti-angiogentic mediators activity.

In PV, the PIGF, another member of VEGF family, seems to be of special interest. Its main receptor, the VEGFR-1, is known to be a negative angiogenesis regulator preventing the VEGF binding to the VEGFR-2, indispensable for proangiogenic effect. Increased angiogenesis stimulates proliferation of anti-angiogenic factors, including endostatin, which counteracts the endothelial cells' proliferation and migration stimulated by the VEGF. What is more, endostatin increases the recruitment of other anti-angiogenic mediators, including thrombospondins andglicoproteins which, among others, increase adhesion of the endothelial cells $[19,20]$.

The results of our study are in accordance with the observations presented by other authors who reported differences between the PV and PsA NVC images. They found that twisted and dilated venular branches [21] or coiled, tortuous and short capillaries [22,23] might be characteristic NVC features in PV, whereas a reduction in the capillaries linear density [24], lower mean capillary length [25], a decreased diameter of afferent limb and meandering capillaries with tight terminal convolutions [26] were characteristic of PsA. Similarly to our observations, the well visible sub-papillary venular plexus could turn out to be another distinctive feature while distinguishing between PsA and PV [27,28]. According to some of the authors, the NVC pattern may also be dependent on the nail plate involvement or PsA subtype, i.e. a diminution in the capillary bed density in the nailfold of psoriatic patients with associated nail changes or DIP (distal interphalangeal predominant) joint disease [24]. The same authors were also of the opinion that NVC could be helpful in differentiation between PsA and rheumatoid arthritis (RA) in which the venous, arterial and loop diameters were significantly higher than in PV, PsA and controls [29].

On the basis of the results of our NVC examination we were able to distinguish four original types of NVC patterns in psoriatic patients (Table 2, Figure 3). Interestingly, we found a significant correlation between the NVC pattern and PsA. Type IV of NVC pattern, however, was not observed in the patients with no joint involvement. In the PV patients, the Type I and Type II NVC patterns were more frequent in comparison with the patients with PsA [30]. No correlations between NVC pattern and serum activity of angiogenic markers were revealed.

Alopecia areata and androgenetic alopecia. Hair follicle angiogenesis is a complex process controlled by both proangiogenic and antiangiogenic factors [31-33]. Among
TABLE 2. Types of capillaroscopic patterns in psoriatic patients.

\begin{tabular}{lc}
\hline \hline Type & Capillaroscopic pattern \\
\hline 1 & $\begin{array}{c}\text { Normal or close to normal, with pink or pale pink background } \\
\text { and few tortuous capillaries }\end{array}$ \\
\hline 2 & Many tortuous, coiled, ramiefied or "M" shaped capillaries \\
\hline 3 & Red background, oedema and "plaque-type" capillaries \\
\hline 4 & $\begin{array}{r}\text { Pale background, decreased number of capillaries } \\
\text { and/or presence of frail and thin capillaries } \\
\text { and well-visible subpapillaryvenular plexus. }\end{array}$ \\
\hline
\end{tabular}

different mediators under special interest are: VEGF-A, HGF, IGF-1. These factors exert not only proangiogenic influence but they are also involved in hair cycle control. Together with VEGF, HGF and IGF-1, they are considered to be crucial for anagen maintance [34].

It has been demonstrated that increased expression of VEGF in hair follicle keratinocytes is associated with increased vascularisation of hair follicle, which is characteristic for anagen. A reduced vasculature around hair follicle is related to decreased synthesis of VEGF [32,33]. Interestingly enough, HGF and IGF-1 exert stimulatory effect on VEGF expression. Therefore, it may be expected that these factors may be also involved in anagen-associated angiogenesis $[33,34]$. In the group of antiangiogenic factors endostatin deserves special attention. It has been suggested that this potent endogenic inhibitor of pathogenesis might be involved in the control of hair cycle-associated angiogenesis. However, its role has yet to be clarified [35]. It is believed that any alterations in microcirculation should be noticeable in the nailfold, therefore NVC might be used in all clinical conditions in which microcirculation involvement is expected [36].

Up-to-date capillaroscopic abnormalities in AA have been reported only in a few studies [37,38], however no typical pattern has been identified yet. Thus, our findings are difficult to interpret. Nevertheless, the presence of various NVC alterations may suggest disturbances in skin microcirculation in the course of AA.

To the best of our knowledge, NVC has never been used to observe microcirculation in androgenetic alopecia. Observed alterations are not characteristic, but they may suggest angiogenesis. The lack of other studies considering NVC in androgenetic alopecia, makes it difficult to interpret. However, the possibility that more prominent changes might be observed within the scalp cannot be also excluded

Also, it is possible that the observed NVC alterations in both investigated types of non-scaring alopecia may result from local, rather than systemic disturbances in angiogenesis control.

\section{CONCLUSIONS}

1. Increased levels of Ang-2 in serum of SSc patients with late capillaroscopic pattern might be associated with advanced capillary damage

2. The inverse correlation between total levels of EMPs with the severity of capillary loss may reflect microvascular changes in SSc patients

3. The obtained nailfold pattern may be helpful in the differentiation between PsA and PV 
4. The presence of various NVC patterns among AA and androgenetic alopecia patients may suggest disturbances in skin microcirculation in course of the diseases

5. Further studies are required to fully elucidate observed correlations and their clinical significance.

\section{REFERENCES}

1. Cutolo M. Atlas of Capillaroscopy in rheumatic diseases. 1st ed. Milano: Elsevier; 2010.

2. Gallucci F, Russo R, Buono R, et al. Indications and results of videocapillaroscopy in clinical practice. Adv Med Sci. 2008;53(2):1-9.

3. Jimenez JJ, Jy W, Mauro LM, et al. Endothelial microparticles (EMP) as vascular disease markers. Adv Clin Chem. 2005;39:131-57.

4. Bernal-Mizrachi L, Jy W, Fierro C, et al. Endothelial microparticles correlate with high-risk angiographic lesions in acute coronary syndromes. Int J Cardiol. 2004;97(3):439-46.

5. Michalska-Jakubus M, Kowal-Bielecka O, Chodorowska G, et al. Angiopoietins- 1 and -2 are differentially expressed in the sera of patients with systemic sclerosis: high angiopoietin-2 levels are associated with greater severity and higher activity of the disease. Rheumatol. 2011;50(4):74655.

6. Gerkowicz A, Krasowska D, Pietrzak A, et al. Videocapillaroscopic alterations in alopecia areata. Biomed Rese Int. 2013;2013:160203.

7. Brown GE, O'Leary PA. Skin capillaries in scleroderma. Arch Intern Med. 1925;36:73-88.

8. Maricq HR, LeRoy EC. Patterns of finger capillary abnormalities in connective tissue disease by "wide-field" microscopy. Arthritis Rheum. 1973;16(5):619-28.

9. Cutolo M, Sulli A, Pizzorni C, Accardo S. Nailfold videocapillaroscopy assessment of microvascular damage in systemic sclerosis. J Rheumatol. 2000;27(1):155-60.

10. Minier T, Guiducci S, Bellando-Randone S, et al. Preliminary analysis of the very early diagnosis of systemic sclerosis (VEDOSS) EUSTAR multicentre study: evidence for puffy fingers as a pivotalsign for suspicion of systemic sclerosis. Ann Rheum Dis. 2014;73(12):2087-93.

11. van den Hoogen F, Khanna D, Fransen J, et al. 2013 classification criteria for systemic sclerosis: an American College of Rheumatology/ European League against Rheumatism collaborative initiative. Arthritis Rheum. 2013;65(11):2737-47.

12. Sebastiani M, Manfredi A, Colaci M, et al. Capillaroscopic skin ulcer risk index: a new prognostic tool for digital skin ulcer development in systemic sclerosis patients. Arthritis Rheum. 2009;61(5):688-94.

13. Riccieri V, Vasile M, Iannace N, et al. Systemic sclerosis patients with and without pulmonary arterial hypertension: a nailfold capillaroscopy study. Rheumatology. 2013;52(8):1525-8.

14. Castellví I, Simeón-Aznar CP, Sarmiento M, et al. Association between nailfold capillaroscopy findings and pulmonary function tests in patients with systemic sclerosis. J Rheumatol. 2015;42(2):222-7.

15. Yancopoulos GD, Davis S, Gale NW, et al. Vascular-specific growth factors and blood vessel formation. Nature. 2000;407:242-8.

16. Dignat-George F, Boulanger CM. The many faces of endothelial microparticles. Arterioscler Thromb Vasc Biol. 2011;31(1):27-33.
17. Braverman IM, Yen A. Ultrastructure of the capillary loops in the dermal papillae. J Invest Dermatol. 1977;68:53-60.

18. Micali G, Lacarrubba F, Musumeci ML, et al. Cutaneous vascular patterns in psoriasis. Int J Dermatol. 2010;49:249-56.

19. Liew SC, Das-Gupta E, Chakravarthi S, et al. Differential expression of the angiogenesis growth factors in psoriasis vulgaris. BMC Res Notes. 2012;5:201.

20. Batycka-Baran A, Krawczenko A, Paprocka M, et al. The soluble vascular endothelial growth factor receptor-1 (sVEGFR-1) in patients with plaque psoriasis - does it play any role? Cytokine. 2013;61(2):6889.

21. Ramos-e-Silva M, Bernardini E, Filgueira AL. Capillaroscopy in psoriasis vulgaris. J Eur Acad Dermatol Venereol. 1996;7:192-3.

22. Ohtsuka T, Yamakage A, Miyachi Y. Statistical definition of nailfold capillary pattern in patients with psoriasis. Int J Dermatol. 1994;33:77982.

23. Leroux B, Barraza S, Estrella V, et al. Valor clínico de las imágenes de capilaroscopia periungueal en pacientes con psoriasis. Med Cutan Iber Lat Am. 2004;32:61-4.

24. Bhushan M, Moore T, Herrick AL, Griffiths CEM. Nailfold video capillaroscopy in psoriasis. Br J Dermatol. 2000;142:1171-6.

25. Salli L, Raimondi F, Pappalardo A. Periungual capillaroscopy in psoriatic arthritis. Clin Ter. 1999;150:409-12.

26. Redisch W, Messina EJ, Hughes G, McEwen C. Capillaroscopic observations in rheumatic diseases. Ann Rheum Dis. 1970;29:244-53.

27. Zaric D, Clemmensen OJ, Worm AM, Stahl D. Capillary microscopy of the nail fold in patients with psoriasis and psoriatic arthritis. Dermatologica. 1982;164:10-4.

28. Cervini C, Grassi W. Periungual capillary microscopy in patients with chronic polyarthritis and psoriasis arthropathica. Z Rheumatol. 1984;43:311-3.

29. Graceffa D, Amorosi B, Maiani E, et al. Capillaroscopy in psoriatic and rheumatoid arthritis: a useful tool for differential diagnosis. Arthritis. 2013;2013:957480.

30. Bartosińska J, Chodorowska G. Autorska propozycja klasyfikacji obrazów kapilaroskopowych w łuszczycy zwyczajnej i stawowej. Post Dermatol Alergol. 2009;26(1):17-24.

31. Yano K, Brown LF, Detmar M. Control of hair growth and follicle size by VEGF-mediated angiogenesis. J Clin Invest. 2001;107(4):409-17.

32. Simonetti O, Lucarini G, Bernardini ML, et al. Expression of vascular endothelial growth factor, apoptosis inhibitors (survivin and p16) and CCL27 in alopecia areata before and after diphencyprone treatment: an immunohistochemical study. Br J Dermatol. 2004;150:940-8.

33. Mecklenburg L, Tobin DJ, Müller-Röver S, et al. Active hair growth (anagen) is associated with angiogenesis. J Invest Dermatol. 2000;114:90916.

34. Schneider MR, Schmidt-Ullrich R, Paus R. The hair follicle as a dynamic miniorgan. Curr Biol. 2009;19(3):R132-42.

35. Miele C, Rochford JJ, Filippa N, et al. Insulin and insulin-like growth factor-I induce vascular endothelial growth factor mRNA expression via different signaling pathways. J Biol Chem. 2000;275:21695-702.

36. Grassi W, Del Medico P. Atlas of capillaroscopy. Milano: Medical Publishing and New Media EDRA; 2004.

37. Ganzetti G, Campanati A, Simonetti O, et al. Videocapillaroscopic pattern of alopecia areata before and after diphenylcyclopropenone treatment. Int J Immunopathol Pharmacol. 2011;24:1087-91.

38. Rubisz-Brzezińska J, Bendkowski W. Capillaroscopic pattern of ungual folds of the fingers in alopecia areata. Przegl Dermatol. 1979;66:471-5.

\section{Corresponding author}

Michał Adamczyk

13 Radziwiłłowska Str., 20-080 Lublin, Poland

Tel. (fax): 81 532-36-47

E-mail: michaladamczyk1310@wp.pl

Photographic documentation supplied by the Authors. 